\title{
Sedoxantrone Trihydrochloride
}

National Cancer Institute

\section{Source}

National Cancer Institute. Sedoxantrone Trihydrochloride. NCI Thesaurus. Code C1576.

The trihydrochloride salt of the anthrapyrazole antineoplastic antibiotic sedoxantrone with potential antineoplastic activity. Sedoxantrone intercalates into DNA and interacts with topoisomerase II, thereby inhibiting DNA replication and repair and RNA and protein synthesis. 